\title{
Optimization of culture conditions for mycelial growth and basidiocarp production of Philippine strains of Panaeolus antillarium and Panaeolus cyanescens
}

\author{
Bustillos RG ${ }^{1 *}$, Dulay $\mathbf{R M R}^{2,3}$, Kalaw $\mathrm{SP}^{2,3}$ and Reyes $\mathbf{R G}^{2,3}$ \\ ${ }^{l}$ Nueva Ecija University of Science and Technology, Cabanatuan City, Nueva Ecija, Philippines \\ ${ }^{2}$ Center for Tropical Mushroom Research and Development, Central Luzon State University, Science City of Munoz, \\ Nueva Ecija, Philippines \\ ${ }^{3}$ Department of Biological Sciences, College of Arts and Sciences, Central Luzon State University, Science City of \\ Munoz, Nueva Ecija, Philippines
}

Bustillos RG, Dulay RMR, Kalaw SP, Reyes RG 2014 - Optimization of culture conditions for mycelial growth and basidiocarp production of Philippine strains of Panaeolus antillarium and Panaeolus cyanescens. Mycosphere 5(3), 398-404, Doi 10.5943/mycosphere/5/3/1

\begin{abstract}
The domestication and optimization of cultural conditions for the secondary mycelial growth was determined to establish the production technology of Panaeolus species. Wild $P$. antillarum and $P$. cyanescens were collected and their cell lines were rescued. The mycelial growth performance was evaluated on different indigenous culture media (carabao, cow and horse dung) and physical factors ( $\mathrm{pH}$, aeration and illumination). The optimal growth condition for basidiocarp production using dung of domesticated ruminants was also determined. Both secondary mycelia of the two Panaeolus species evaluated grew best on solid culture media of carabao and cow dung decoction gulaman (local crude agar) at a $\mathrm{pH}$ range of 7.5 - 8.0, incubated in unsealed and dark condition. Carabao and cow dung as substrates favored the fast mycelial colonization of both mushrooms with means of $11.20 \pm 4.24$ and $11.50 \pm 5.80$ days, respectively. However, regardless of the substrate, $P$. antillarum colonized the substrates in a shorter period of $9.37 \pm 2.74$ days. In terms of yield, carabao dung had the highest mean yield of $7.16 \pm 0.75 \mathrm{~g}$ with $17.89 \pm$ $1.88 \%$ bio-efficiency, which did not significantly vary with cow dung having a mean yield of 6.99 $\pm 1.89 \mathrm{~g}$ with $17.49 \pm 4.73 \%$ bio-efficiency. These significant results suggest that $P$. antillarum and $P$. cyanescens are new Philippine strains of wild mushrooms with great potential for cultivation for nutraceutical purposes.
\end{abstract}

Key words - Panaeolus species - hallucinogenic mushrooms - secondary mycelia - basidiocarp, coprophilous mushrooms

\section{Introduction}

Mushrooms have long been prized around the world for their extraordinary medicinal and immune-enhancing and nutraceutical qualities. Medicinal mushrooms are usually consumed as hot water extracts, concentrates, powder, health tonics, tea, soup and herbal formulation (Chang 2008). For decades, clinical medicine has become successful in isolating important compounds that have shown promising immunomodulatory, antitumor, antiviral, antibacterial, and anti-diabetic 
properties (Wasser 2002). Using the mycelium and basidiocarp extracts of the mushroom distinguishes nutraceutical, food or a drug (Chang 2001).

The Philippines harbors different edible species that have been used for medicinal purposes. Few attentions have been given to hallucinogenic mushrooms due to reported abuse as drugs and known to contain toxic components. The group of hallucinogenic mushrooms (species of the genera Conocybe, Gymnopilus, Panaeolus, Pluteus, Psilocybe, and Stropharia) is psilocybin-containing mushrooms. These psychoactive mushrooms contain psilocybin and its active metabolite psilocin is similar to lysergic acid diethylamide and the effect of serotonin (Reingardiene et al. 2005). These hallucinogens also affect the central nervous system rapidly producing ataxia, hyperkinesis, and hallucinations (Reingardiene et al. 2005).

Reports of toxicity associated with this group of mushrooms have increased because of their growing popularity as hallucinogens. Genus Panaeolus belongs to the group of hallucinogenic mushrooms. It is a relatively small genus of black spored little brown mushrooms with a bell shaped to conical cap and thin, brittle stalk. The sides of the gills often have a mottled or spotted appearance due to uneven maturation of the spore producing cells (basidia), but they do not deliquesce (a process of autodigestion whereby the cap is reduced to a black liquid) as in Coprinus. Psilocybe and Conocybe are common in dung, but do not have black spores. Panaeolus is abundant in pastures, lawns, and manure heaps, fruiting whenever it's moist (Stamets 1996). These psychoactive mushrooms occur in all continents, but the majority of species are found in subtropical humid forests (Guzman et al. 2000).

Given the less attention due to their hallucinogenic effects, our team aimed to develop production technology of Panaeolus species with great potential in the nutraceutical and pharmaceutical industry.

\section{Methods}

\section{Source of strains}

Wild fruiting bodies of Panaeolus species were collected in the pasture lands of Lupao, Nueva Ecija. These were placed in a polypropylene plastic and brought in the laboratory of the Center for Tropical Mushroom Research and Development to rescue their mycelia following the standard protocol of mushroom tissue culture (Reyes et al. 1998). Coconut water gulaman was used as the culture medium. Cultures were incubated at room temperature to allow mycelia growth. These were served as stock culture and used as source of inocula in the evaluation of the optimum requirements for efficient mycelia growth.

\section{Influence of the nutritional factors}

The following locally available culture media were formulated and evaluated for the mycelial growth of Panaeolus spp.: $\mathrm{T}_{1}-1$ liter decoction from $50 \mathrm{~g}$ carabao dung; $\mathrm{T}_{2}-1$ liter decoction from $50 \mathrm{~g}$ cow dung and $\mathrm{T}_{3}-1$ liter from $50 \mathrm{~g}$ horse dung. The three evaluated media were added with $2 \%$ gelatin prior to sterilization. Triplicate plates per medium were aseptically inoculated with a $10 \mathrm{~mm}$ diameter of mycelial disc of Panaeolus spp. and subsequently incubated at room temperature to allow the ramification of its mycelia. Daily mycelial growth diameter was recorded.

\section{Influence of physical factors}

The most appropriate medium in study I was adjusted to different $\mathrm{pH}$ levels: 6.0, 6.5, 7.0, 7.5 and 8.0 using $0.1 \mathrm{M} \mathrm{NaOH}$ or $0.1 \mathrm{M} \mathrm{HCl}$ prior to sterilization. Triplicate plates per medium were aseptically inoculated with a $10 \mathrm{~mm}$ mycelial disc of Panaeolus spp. and subsequently incubated at room temperature to allow the ramification of its mycelia. Daily mycelial growth was recorded.From the most appropriate medium and $\mathrm{pH}$ level, the influence of aeration on the mycelial growth of Panaeolus spp. was investigated. This was done by sealing individually the triplicate plates with parafilm $\left(\mathrm{T}_{1}\right)$ and the remaining triplicates were not sealed $\left(\mathrm{T}_{2}\right)$. The plates were 
incubated at room temperature to allow the proliferation of mycelia. Daily mycelial growth was recorded. Six plates containing the most appropriate medium were adjusted to the optimum $\mathrm{pH}$ level and inoculated with $10 \mathrm{~mm}$ mycelial disc of Panaeolus spp. and incubated in two different aeration conditions (sealed or unsealed). After determining the most appropriate aeration condition, inoculated plates were incubated into two illumination conditions (dark and lighted). Daily mycelial growth diameter was recorded.

\section{Basidiocarp Production}

The fruiting body production of Panaeolus spp. was evaluated on the following formulations: carabao dung, cow dung, and horse dung. Each substrate (40 g) was placed in clear bottle and individually covered with polypropylene sheets prior to sterilization at $15 \mathrm{psi}$ or $121^{\circ} \mathrm{C}$ for 45 minutes. After cooling, the sterilized bottled substrates were inoculated with a $10 \mathrm{~mm}$ diameter of mycelial disc of Panaeolus spp. and subsequently incubated at room temperature. Incubation period and weight of fruiting bodies were determined.

Data were analyzed using Analysis of Variance (ANOVA) and means were compared using Least Significant Difference (LSD).

\section{Results and Discussion}

\section{Influence of Nutritional Factors}

Mycelia are a fabric of interconnected interwoven strands of cells. In mushroom production, mycelia stock culture is a very important source of mushroom cell lines (Dulay et al. 2012). The luxuriance and rapidity of growth of a certain mushroom partly depend on the appropriate culture medium used in its cultivation in the laboratory (Reyes et al. 2009). The growth of mycelia is influenced by the nutritional content of the organic matter of the substrates. Nutritional evaluation is therefore necessary to determine the best medium which favors the efficient growth of mushroom species. In this study, three indigenous culture media were used to evaluate the growth of two species of Panaeolus such as carabao dung gulaman, cow dung gulaman and horse dung gulaman.

The mycelial growth response of two species of Panaeolus on different culture media after 9 days of incubation is presented in Table 1 . It can be noted that $P$. antillarum registered significantly wider mycelial diameter with a mean of $80.54 \pm 2.22 \mathrm{~mm}$ which is statistically different from $P$. cyanescens that registered smaller mycelial growth diameter of $76.46 \pm 7.53 \mathrm{~mm}$. Among the different culture media evaluated, carabao dung gulaman produced the widest mycelia diameter with a mean of $81.37 \pm 0.43 \mathrm{~mm}$ but statistically comparable to cow dung gulaman with a mean diameter of $81.17 \pm 1.82 \mathrm{~mm}$. Cow dung gulaman favored the efficient colonization of mycelia of $P$. antillarum and $P$. cyanescens within 9 days of incubation producing a dense cottony mycelial growth with zonation patterns (Fig. 1). On the other hand, horse dung gulaman registered significantly smallest mycelial diameter with a mean of $72.97 \pm 7.26 \mathrm{~mm}$, after nine days of incubation. Mycelial colonization in horse dung gulaman was thin and less aggressive suggesting that the nutrient content of the culture medium might not be sufficient to nourish a vigorous and luxuriant mycelial growth. This response further implies that carabao and cow dung gulaman were favorable growing media for mycelial growth of the two Panaeolus species evaluated. The rapid growth of mycelia of Panaeolus spp. in cow and carabao dung media could be attributed to their nutritional composition. Wattiaux and Howard (2000) reported that cattle dung is rich in minerals and from the highly cellulose digested and undigested fecal residues.

\section{Influence of Physical Factors}

Since cow dung gulaman and carabao dung gulaman respectively showed the most efficient growth of mycelia of $P$. antillarum and $P$. cyanescens, these media were used in the evaluation of physical growth conditions such as $\mathrm{pH}$, aeration and illumination.

In terms of the $\mathrm{pH}$, regardless of the $\mathrm{pH}$ of the media, both species showed fast mycelial growth with a diameter of $76.59 \pm 2.94 \mathrm{~mm}$ for $P$. antillarium, which is statistically comparable to 
Table 1 Influence of nutritional and physical factors on the mycelial growth of Panaeolus spp. respectively after 9 days and 8 days of incubation.

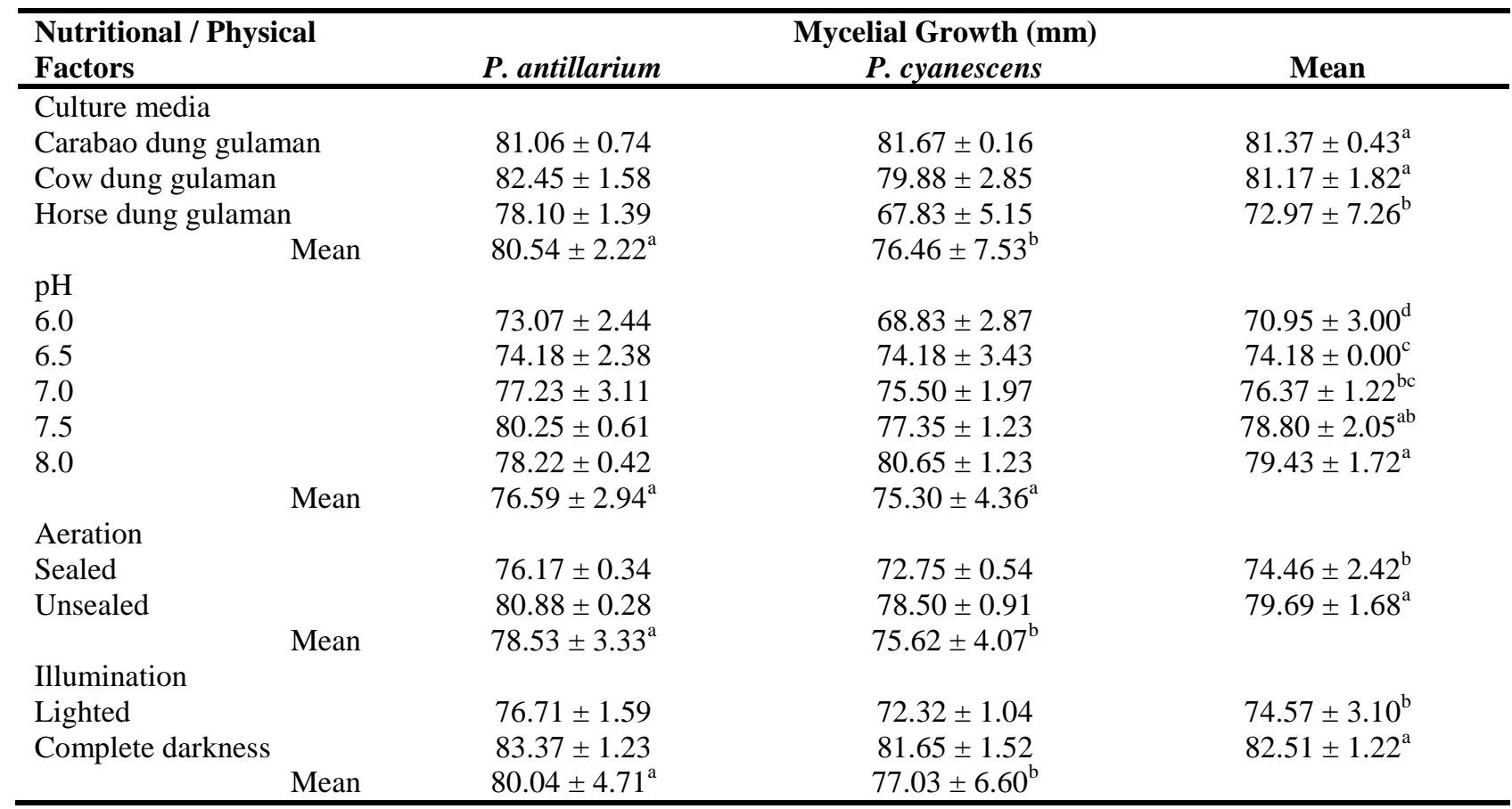

(In Table: Values are the means \pm SD of mycelial growth diameter, and means having the same letter of superscript in the same column are insignificantly different from each other at 5\% level of significance using LSD.)

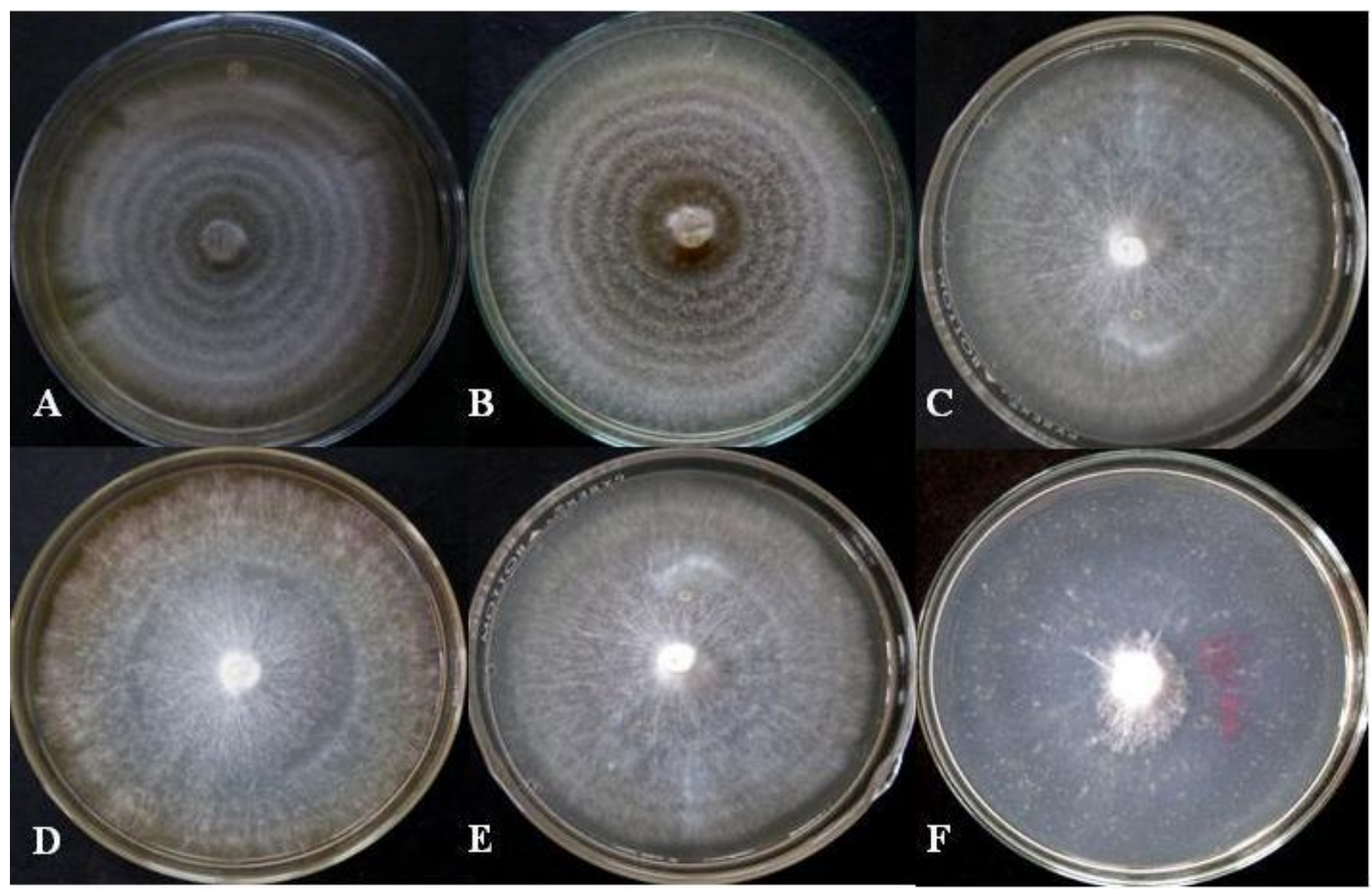

Fig. 1 - Plate culture of $P$. antillarium on (A) carabao dung gulaman, (B) cow dung gulaman, and (C) horse dung gulaman, and $P$. cyanescens on (D) carabao dung gulaman, (E) cow dung gulaman, and $(F)$ horse dung gulaman after 9 days of incubation. 
Table 2 Fruiting body production of Panaeolus species on the different dried dung.

\begin{tabular}{|c|c|c|c|}
\hline Substrate & P. antillarium & P. cyanescens & Mean \\
\hline \multicolumn{4}{|l|}{ Incubation Period (day) } \\
\hline Carabao dung & $8.20 \pm 0.84$ & $14.20 \pm 0.45$ & $11.20 \pm 4.24^{\mathrm{a}}$ \\
\hline Cow dung & $7.40 \pm 0.55$ & $15.60 \pm 1.82$ & $11.50 \pm 5.80^{\mathrm{a}}$ \\
\hline Horse dung & $12.50 \pm 7.07$ & $20.50 \pm 11.2$ & $16.50 \pm 5.66^{\mathrm{b}}$ \\
\hline Mean & $9.37 \pm 2.74^{\mathrm{b}}$ & $16.77 \pm 3.31^{\mathrm{a}}$ & \\
\hline \multicolumn{4}{|l|}{ Weight of fruiting bodies (g) } \\
\hline Carabao dung & $6.63 \pm 3.44$ & $7.69 \pm 2.38$ & $7.16 \pm 0.75^{\mathrm{a}}$ \\
\hline Cow dung & $8.34 \pm 4.67$ & $5.66 \pm 3.42$ & $6.99 \pm 1.89^{\mathrm{a}}$ \\
\hline Horse dung & $1.02 \pm 1.53$ & $0.95 \pm 1.56$ & $0.98 \pm 0.05^{\mathrm{b}}$ \\
\hline Mean & $5.33 \pm 3.83^{\mathrm{a}}$ & $4.77 \pm 3.46^{\mathrm{a}}$ & \\
\hline \multicolumn{4}{|l|}{ Biological efficiency (\%) } \\
\hline Carabao dung & $16.56 \pm 8.61$ & $19.22 \pm 5.94$ & $17.89 \pm 1.88^{\mathrm{a}}$ \\
\hline Cow dung & $20.84 \pm 11.7$ & $14.15 \pm 8.55$ & $17.49 \pm 4.73^{\mathrm{a}}$ \\
\hline Horse dung & $2.55 \pm 4.44$ & $2.37 \pm 3.91$ & $2.46 \pm 0.13^{b}$ \\
\hline Mean & $13.32 \pm 9.57^{\mathrm{a}}$ & $11.91 \pm 8.64^{\mathrm{a}}$ & \\
\hline
\end{tabular}

(In Table: Means having the same letter of superscript in the same column are insignificantly different from each other at $5 \%$ level of significance using LSD.)

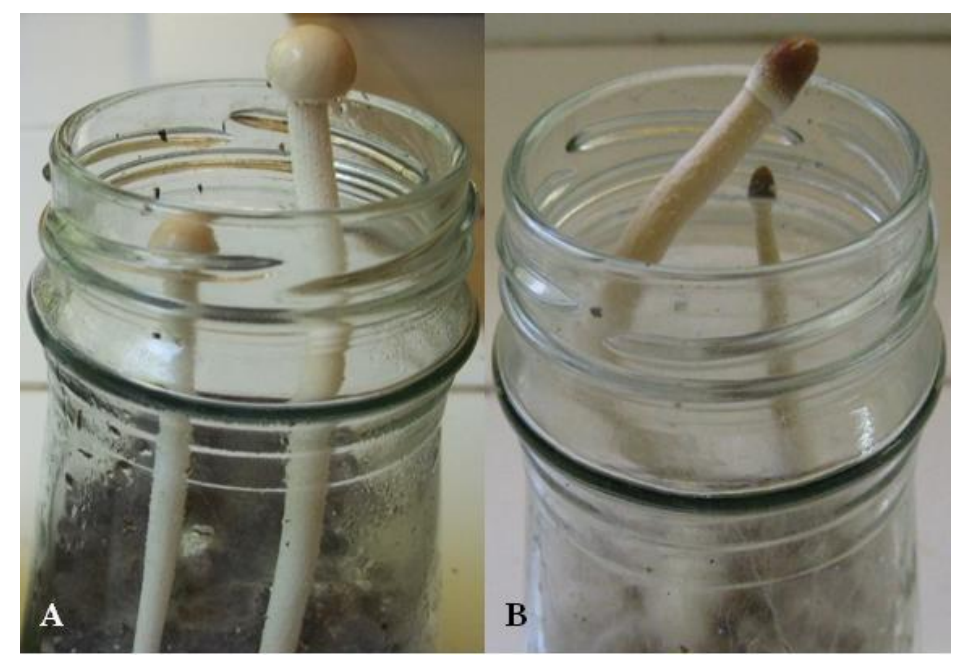

Fig. 2 - Fruiting bodies of $P$. antillarium (A) and P. cyanescens (B) grown on carabao dung in miniaturized bottles.

$75.30 \pm 4.36 \mathrm{~mm}$ diameter of mycelial growth of $P$. cyanescens. Among the different $\mathrm{pH}$ levels evaluated, $\mathrm{pH} 8.0$ recorded the widest mycelial diameter of $79.43 \pm 1.72 \mathrm{~mm}$. $P$. antillarum and $P$. cyanescens favorably grow in medium with $\mathrm{pH} 8.0$ producing a uniform white cottony and vigorous mycelial growth with zonation patterns on the 8th day of incubation. However, $\mathrm{pH} 6.0$ had the lowest mycelia growth with a mean diameter of $70.95 \pm 3.00 \mathrm{~mm}$. Xin-Sheng (2005) reported that the optimum $\mathrm{pH}$ requirement for efficient mycelia growth of P. bisporus is within $\mathrm{pH}$ 6.5-8.0.

After establishing the optimum $\mathrm{pH}$ of cow and carabao dung media, the influence of aeration in the mycelial growth of Panaeolus was investigated. It can be seen that the wider mycelial diameter was observed in unsealed plates with a mean of $79.69 \pm 1.68 \mathrm{~mm}$ compared to those incubated in sealed condition with a diameter growth of $74.46 \pm 2.42 \mathrm{~mm}$. This result suggests that aeration is another important physical factor to be considered for efficient mycelial growth of the two Panaeolus species. The unsealed plates showed thick mycelia with complete mycelia ramification for 8 days.

Another physical requirement evaluated is the influence of illumination on the mycelial growth of Panaeolus. Plate cultures incubated in dark conditions significantly stimulated mycelial growth with a diameter of $82.51 \pm 1.22 \mathrm{~mm}$ than those incubated in lighted condition with $74.57 \pm$ 
$3.10 \mathrm{~mm}$. Regardless of the two conditions, $P$. antillarium significantly registered wider mycelial diameter with a mean of $80.04 \pm 4.71 \mathrm{~mm}$ while $P$. cyanescens had $77.03 \pm 6.60 \mathrm{~mm}$. This result indicates that $P$. antillarum and $P$. cyanescens favorably grow in dark condition. Similar responses were observed with $C$. reinakeana (Reyes et al. 1998), C. comatus (Reyes et al. 2009) and $L$. tigrinus (Dulay et al. 2012) where dark condition is the required illumination condition.

\section{Basidiocarp Production}

The established optimal requirements of secondary mycelia of the two Panaeolus species served as the baseline information in developing a successful production technology. In order to develop a favorable production for mushrooms it is also important to understand their natural habitat, environmental and physiological requirements (Dulay et al. 2012).

In this study, Panaeolus species were grown on different indigenous substrates such as carabao dung, cow dung and horse dung. Mushroom substrate structure has been reported to be an important factor for the growth of mycelium as it allows penetration of mycelium, which ultimately influences fruiting (Tripathy et al. 2011). Also, mushrooms have extensive enzyme systems capable of utilizing organic compounds which occurs in the substrates (Mane et al. 2007).

The number of days of the total incubation period of Panaeolus species is depicted in Table 2. $P$. antillarum significantly recorded the shorter incubation period with a mean of $9.37 \pm 2.74$ days compared with $P$. cyanescens with $16.77 \pm 3.31$ days. Regardless of the species, both species cultivated in carabao dung recorded the shortest day of incubation with $11.20 \pm 4.24$ days, which did not significantly differed with the cow dung with $11.50 \pm 5.80$ days. Further, those incubated in horse dung significantly registered the most extensive incubation period with a mean of $16.50 \pm$ 5.66 days. This result suggests that carabao and cow dung are favorable substrates than the horse dung. This result conforms to the report of Allen (1994) that Panaeolus subbalteatus when cultivated in pure dried carabao dung registers 7-12 days of incubation period. Moreover, substrate supplemented with cow dung completes the mycelial colonization of Coprinus cinereus for 8-12 days.

After the successful mycelia colonization in the entire substrate this is followed by the emergence of the fruiting bodies, which dictates the successful production technology. The weight of fruiting bodies and biological efficiency were presented in Table 2. Significant differences were found in mean weight of fruiting bodies and bio-efficiency among the different dung substrates but not on the two species. It can be noted that $P$. antillarium had higher mean yield with $5.33 \pm 3.83 \mathrm{~g}$ than $P$. cyanescens with $4.77 \pm 3.46 \mathrm{~g}$. The healthy fruiting bodies of the two species of Panaeolus are shown in Fig 2. On the other hand, the highest mean yield was recorded in carabao dung having $7.16 \pm 0.75 \mathrm{~g}(17.89 \pm 1.88 \%$ bio-efficiency), which found not significantly different to cow dung with $6.99 \pm 1.89 \mathrm{~g}(17.49 \pm 4.73 \%$ bio-efficiency). No fruiting bodies were harvested in other replicates of horse dung which significantly recorded the lowest bio-efficiency of $2.46 \pm 0.13 \%$. These results of fruiting body production could be attributed to the components of the different ruminants dung as substrates. The resultant fecal matter produced by cow and carabao after digestion is rich in minerals, debris from cells within the digestive tract. These variations could affect the growth and yield of Panaeolus. Moreover, water holding capacity, interaction between the environmental factors and the nutrients of the substrate has been reported as factors to play important role in inducing formation of fruiting bodies (Reyes et al. 2009, Kurtzman et al. 2010).

Altogether, these established data on the successful production technology suggest that $P$. antillarium and $P$, cyanescens are another additions to the new record of domesticated wild mushrooms in the Philippines. Cultivation must be intensified in order to realize its full pharmaceutical potential. Two types of studies are recommended- first is enrichment of its $\mathrm{C}: \mathrm{N}$ ratio to improve its biological efficiency and second, the functional activities must also be undertaken.

\section{Acknowledgements}

This study was supported by a thesis grant from the Commission on Higher Education in the Philippines. The authors wish to thank the Mushroom Center staffs and the Nueva Ecija University of Science and Technology family for their endless support. 


\section{References}

Allen J, Gartz J, Merlin D. 1994 - Ethnomycology, biochemistry, and cultivation of Psilocybe samiensis Guzman, Bandala and Allen, a new psychoactive fungus from KohSameii, Thailand. Journal of Ethnopharmacology 43, 73-80.

Chang ST. 2001 - A 40-year journey through bioconversion of lignocellulosic wastes to mushrooms and dietary supplements. International Journal of Medicinal Mushrooms 3, 299310.

Chang ST. 2008 - Products of Medicina Mushrooms as a Good Source of Dietary Supplements. Retrieved on December 2011 at http://www.isms.biz/article2.htm.

Dulay RMR, Kalaw SP, Reyes RG, Cabrera AC, Alfonso NF. 2012 - Optimization of Culture Conditions for Mycelial Growth and Basidiocarp Production of Lentinus tigrinus (Bull.) Fr., A new Record of domesticated wild edible mushroom in the Philippines. Philippines Agricultural Scientist 95(3), 209-214.

Guzman G, Allen JW, Gartz J. 2000 - A worldwide geographical distribution of the neurotropic fungi, an analysis and discussion. Annali del MuseoCivico di Rovereto: SezioneArcheologia, Storia, Scienze Naturali 14, 189-280.

Kurtzman RH Jr. 2010 - Ventilation for mushroom cultivation: the importance of the needs of mushrooms and of the gas laws. Micologia Aplicada International 22(2), 63-78.

Mane VJ, Patil SS, Syed AA, Baig MM. 2007 - Bioconversion of low quality lignocellulosic agricultural wastes into edible protein Pleurotus sajor-caju (Fr.) Singer J. Zhejiang. Univ.Sci.8, 745-751.

Reingardiene D Vilcinskaite J, Lazauskas R. 2005 - Hallucinogenic mushrooms. Clinic of Intensive Therapy. Kaunas University of Medicine, Kaunas, Lithuania. 41(12), 1067-1070.

Reyes RG, Eguch F, Iijima T, Higaki M. 1998 - Influence of medium composition and plant growth regulators on the mycelial growth of Collybia reinakeana. Journal of Agricultural Sciences - Tokyo University of Agriculture. 43, 43-50.

Reyes RG, Lopez LLMA, Kumakura, Kalaw SP, Kikukawa TEF. 2009 - Coprinus comatus, a newly domesticated wild nutriceutical mushroom in the Philippines. Journal of Agricultural Technology 5(2), 299-316.

Stamets P. 1996 - Psilocybin Mushrooms of the World. Ten Speed Press. Crown Publishing Group, New York 11-20.

Tripathy A, Sahoo TK, Begera SR. 2011 - Yield evaluation of paddy straw mushrooms (Volvariella spp.) on various lignocellulosic wastes. Botanical Research International 4(2), 19-24.

Wasser SP. 2002 - Medicinal mushrooms as a source of antitumor and immunomodulatory polysaccharides. Applied Microbiology and Biotechnology 60, 258-274.

Wattiaux MA, Howard WT. $2000-$ Digestion in the dairy cow. at http://babcock.cals.wisc.edu/downloads/de/01.en.pdf.

Xin-Sheng HE. 2005 - Physiological characteristics of Panaeolus bisporus. Journal of Microbiology at www. cnki.com - 2011. 